\title{
Improvement of Infraorbital Laxity and Wrinkles by Combined Use of Microfocused Ultrasound and Absorbable Polydioxanone Threads in a Post-Blepharoplasty Patient
}

Nark-Kyoung Rho

Sung Tae Chung

Cheongdam Leaders Dermatology Clinic, Seoul, Korea
There are limitations to the conventional transconjunctival lower orbital fat removal surgery in subjects with severe infraorbital laxity. This may be because the reduction of orbital fat volume does not provide sufficient improvement of age-related skin laxity and wrinkles. Post-surgical adjuvant non-surgical procedures may be required in this subset of patients. This case report describes a successful infraorbital rejuvenation using an approach that combined microfocused ultrasound and absorbable thread in an old post-blepharoplasty patient whose surgical outcome was not satisfactory.

\section{Key words}

Infraorbital fat pads; Infraorbital laxity; Microfocused ultrasound; Polydioxanone thread; Transconjunctival blepharoplasty 


\section{INTRODUCTION}

Lower eyelid fat bulging (LEFB), commonly referred to as 'baggy lower eyelids' is frequently observed in adults as a major sign of aging. 'Transconjunctival orbital fat excision, redistribution, or septal tightening remains a popular and safe technique to treat LEFB; however, over-resection of the orbital fat frequently results in a hollowed-out appearance or exaggeration of lower eyelid skin laxity and wrinkles. ${ }^{2}$ Various surgical and non-surgical options have been tried to overcome limitations of traditional surgical blepharoplasty. ${ }^{1}$ We report our experience of the adjuvant use of microfocused ultrasound and absorbable threads in a patient whose concern was a post-blepharoplasty aggravation of lower eyelid laxity.

\section{CASE REPORT}

A 70-year-old Korean male patient visited our clinic for the bulging and laxity of lower eyelids. Physical examination revealed moderate degree of fat bulging and severe laxity and wrinkles of the lower eyelids (Fig. 1). Bulging orbital fat pads were removed by carbon dioxide laser-assisted transconjunctival lower blepharoplasty. No significant postoperative side effects were seen for a 4-week postoperative period. However, the patient was not satisfied with the result of the procedure because of the little improvement of the lower eyelid sagging. Clinical examination 2 months after the surgery found that the patient's lower eyelid skin laxity and wrinkles have not improved whereas the degree of fat bulging has been

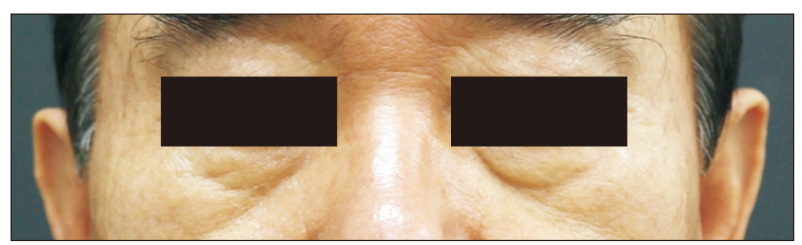

Fig. 1. Lower eyelid fat bulging, laxity, and wrinkles in a 70-year-old male.

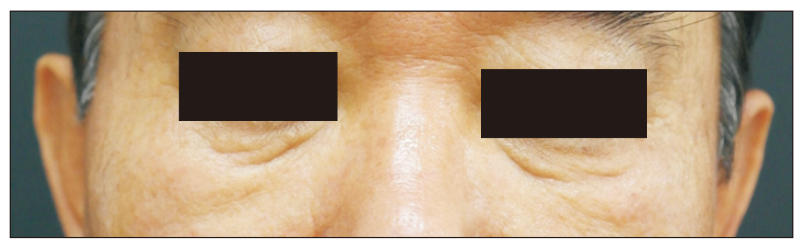

Fig. 2. The patient's lower eyelid skin laxity and wrinkles have not improved whereas the degree of fat bulging has been reduced 2 months after transcutaneous lower orbital fat removal surgery. reduced (Fig. 2). Comparison of the patient's before and after blepharoplasty photographs also revealed only minimal improvement of the lower eyelid winkles. For further improvement, additional non-surgical procedures were performed on the patient's lower eyelid skin.

Microfocused ultrasound (Ulthera System; Ulthera, Inc., Mesa, AZ, USA) treatment was performed on the infraorbital area after 45 minutes of topical anesthetic cream application. First, 25 vertical lines were delivered in the infraorbital area with a $10 \mathrm{MHz} / 1.5 \mathrm{~mm}$ transducer and 25 vertical lines were delivered with a $7 \mathrm{MHz} / 3 \mathrm{~mm}$ transducer. In total, 100 lines of microthermal coagulation zones were delivered to the infraorbital area. The energy for the ultrasound pulse was $0.1 \mathrm{~J}$ with the $1.5 \mathrm{~mm}$ transducer and $0.25 \mathrm{~J}$ with the $3 \mathrm{~mm}$ transducer. During the treatment, the transducer was placed firmly and uniformly on the skin surface to prevent superficial thermal injury. During the procedure, an ultrasound image was shown on the screen, and the orbit, septum, and orbital wall were monitored as a safety measure. After the treatment the ultrasound gel was wiped off and ice packs were applied for 5 minutes.

Right after the microfocused ultrasound treatment, absorbable polydioxanone thread insertion was performed to achieve further improvement of the patient's infraorbital skin laxity. The thread used in the present case was a 40-mm length, 6.0-USP monofilament polidoxanone thread (Dermaspring-Eye ${ }^{\circledR}$; Nfinders, Seoul, Korea) which is equipped to a $30-\mathrm{mm}$ length, 30-gauge microcannula. Local anesthesia using a $2 \%$ lidocaine solution with epinephrine $(1: 100,000)$ was administered to the entry sites and a puncture was made using a 27 gauge needle. Fifteen absorbable threads were inserted through the entry site of each infraorbital area. The threads were inserted in a fan-like pattern and the depth of placement was submuscular layer. Following thread insertion, the lower eyelid was compressed with gauze to prevent swelling and hematoma. During the 2-week posttreatment period, no significant side effects were observed except for mild

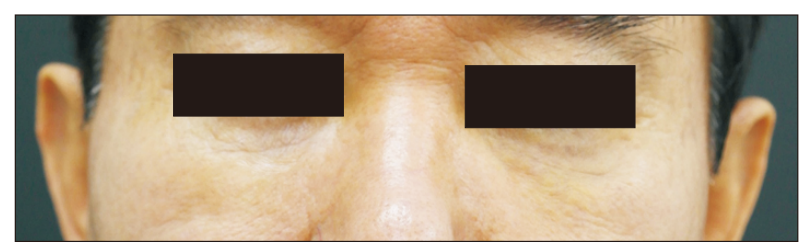

Fig. 3. A significant improvement of infraorbital laxity and wrinkles 10 weeks after combination treatment with focused ultrasound and absorbable polydioxanone threads in the same post-blepharoplasty patient. 
swelling which lasted 3 days.

Ten weeks following the microfocused ultrasound and absorbable thread insertion, the patient showed significant improvement of lower eyelid skin laxity and wrinkles (Fig. 3).

\section{DISCUSSION}

The classical method of correcting lower eyelid fat bulging is blepharoplasty. ${ }^{2}$ However, the latest trend of non-invasive or minimally invasive approaches have introduced combined uses of many nonsurgical procedures with traditional blepharoplasty. ${ }^{1,3}$ Microfocused ultrasound is a new skin tightening treatment modality that offers the potential for non-invasive treatment concepts in dermatology and plastic surgery. ${ }^{4}$ A group of Korean dermatologists assessed the safety and efficacy of microfocused ultrasound for tightening of infraorbital laxity in 15 Korean patients (mean age 50 years) and found out that $87 \%$ of the patients were satisfied with the results of the treatment. ${ }^{4}$ No serious or long-lasting side effects were noted except for mild transient erythema in 2 patients and purpura and edema in 1 patient. Post treatment biopsy specimens showed regenerated and increased collagen and elastic fibers in the reticular dermis. A similar study has been performed by Korean plastic surgeons. ${ }^{3}$ Based on their computed tomography image analysis, microfocused ultrasound treatment has been found to be effective in the mild improvement of lower eyelid fat bulging as well as the skin laxity and texture. In the present case, we performed adjuvant non-invasive microfocused ultrasound on infraorbital area because the patient was not satisfied with the result of traditional transconjunctival lower orbital fat removal surgery in terms of infraorbital laxity and wrinkles. The $1.5 \mathrm{~mm}$ probe was used to tighten of loose eyelid skin and the deep dermis, whereas the $3.0 \mathrm{~mm}$ probe was used to tighten the orbicularis oculi muscle and the orbital septum. ${ }^{3}$

For further improvement, we also inserted absorbable polydioxanone threads in the infraorbital subcutaneous tissue. Currently, absorbable threads are commonly utilized for skin tightening and lifting. A case report from Hwang and the colleagues ${ }^{5}$ has suggested that the polydioxanone threading may be a useful, non-invasive procedure to rejuvenate the aged lower eyelid of patients with anxieties regarding potential complications due to invasive lower blepharoplasty. Recent reports indicate that insertion of absorbable threads into soft tissue induces collagen formation by direct stimulation of fibroblasts and increased cellular activation by converting mechanical stimulation to cellular signaling (mechanotransduction). However, the exact mechanism of action of absorbable threads in the treatment of skin tightening remains unclear.

Our findings suggest that the combination of energybased le.g., microfocused ultrasound, radiofrequency, lasers) and injectable (e.g., absorbable threads, plateletrich plasma, polydeoxyribonucleotide) non-surgical modalities can be an effective and safe post-blepharoplasty procedure in patients with significant infraorbital laxity and wrinkles along with lower eyelid fat bulging. Further clinical trial involving large patient numbers and longterm observation would be necessary to determine the efficacy and safety of the combination treatment.

\section{REFERENCES}

1. Chung ST, Rho NK. Surgical and non-surgical treatment of the lower eyelid fat bulging using lasers and other energy-based devices. Med Lasers 2017;6:58-66.

2. Pacella SJ, Nahai FR, Nahai F. Transconjunctival blepharoplasty for upper and lower eyelids. Plast Reconstr Surg 2010;125:384-92.

3. Pak CS, Lee YK, Jeong JH, Kim JH, Seo JD, Heo CY. Safety and efficacy of ulthera in the rejuvenation of aging lower eyelids: a pivotal clinical trial. Aesthetic Plast Surg 2014;38:861-8.

4. Suh DH, Oh YJ, Lee SJ, Rho JH, Song KY, Kim NI, et al. A intense-focused ultrasound tightening for the treatment of infraorbital laxity. J Cosmet Laser Ther 2012;14:290-5.

5. Hwang UK, Kwon YH, Cho YJ, Kang KJ. Improvement of aged lower eyelid by insertion of polydioxanone threads. J Cosmet Med 2017;1:57-9. 